\title{
Finite element analysis on stable performance of new section of aluminium alloy members under axial compression
}

\author{
Xiumei Zheng ${ }^{a}$ and Zheng Zhang ${ }^{b}$ \\ Department of Civil Engineering, Fujian University of Technology, No.3 Xue Yuan Road, \\ University Town, Fuzhou, Fujian, P.R. China, 350118 \\ azxm573987@163.com, bfrp_creep@sina.com
}

\begin{abstract}
Keywords: aluminium alloy member, new section, stable performance, axial compression, FEA Abstract. For new section of aluminium alloy members there are still not enough researches on the mechanical properties and mechanism of action. The nonlinear finite element analysis (FEA) was carried out to study the mechanical properties of new section of aluminium alloy members under axial loading. In this paper, the method was based on FEA software, ANSYS. And geometric and material non-linearities were incorporated in the finite element model. Then the mechanical properties of new section and $\mathrm{H}$-section were compared. Results show that the stability factors of the new section are between that of the strong axes of $\mathrm{H}$-section and the weak axes of $\mathrm{H}$-section.
\end{abstract}

\section{Introduction}

Aluminium alloy are widely used in new structural material. Because the material with unique properties which high strength-to-weight ratios, good durability and ease of fabrication. The melting point of aluminium is only $600^{\circ} \mathrm{C}$, so it can be easy extrusion. And the extrusion process can produce a profile of arbitrary shape which cannot be obtained by the hot rolling method. A wide variety of cross-section types are available, enabling aluminium alloy to be used efficiently under a wide range of loading conditions. Recently, the studies on axial compressive performance of the common cross-section (such as $\mathrm{H}$-shaped cross-section and rectangular hollow sections) have relatively matured [1-2]. However, the research on aluminium alloy structural components with irregular shaped sections is still lack. In order to give the characteristics of aluminium alloy extrusion molding full play, it is necessary to study on the axial compression performance about some new section of aluminium alloy components.

Usually, it is more difficult to use analytical method to determine the exact ultimate load of various kinds of aluminium alloy axial compression members, especially considering the effect of geometrical non-linearity and material non-linearity, and the components of the residual stress, geometric imperfections, end restraint and the effect of different load conditions. At this time, to obtain the limit load, we need to use numerical method, especially the finite element method.

In order to give full play to the advantages of easy manufacture of aluminum alloy, and avoid the adverse effects of welding, it is meaningful to research and develop the new section which is easy toinstalled and connected. In this paper, the component is uniform along the length, so making it easier for the extrusion molding factory. The rib which the inside and the outside are not only to improve the bearing capacity and improve the stability, but also to provide convenience for installation and connection joints. Construction of the components are consistent in all directions. So the components are convenient for connecting side columns and corner columns. The section was shown by Fig. 1.

This paper built up finite element models to simulate new cross-section of aluminium alloy under axial compression. Then, the results of a new sections and the H-shaped cross section were compared by the FEA. 

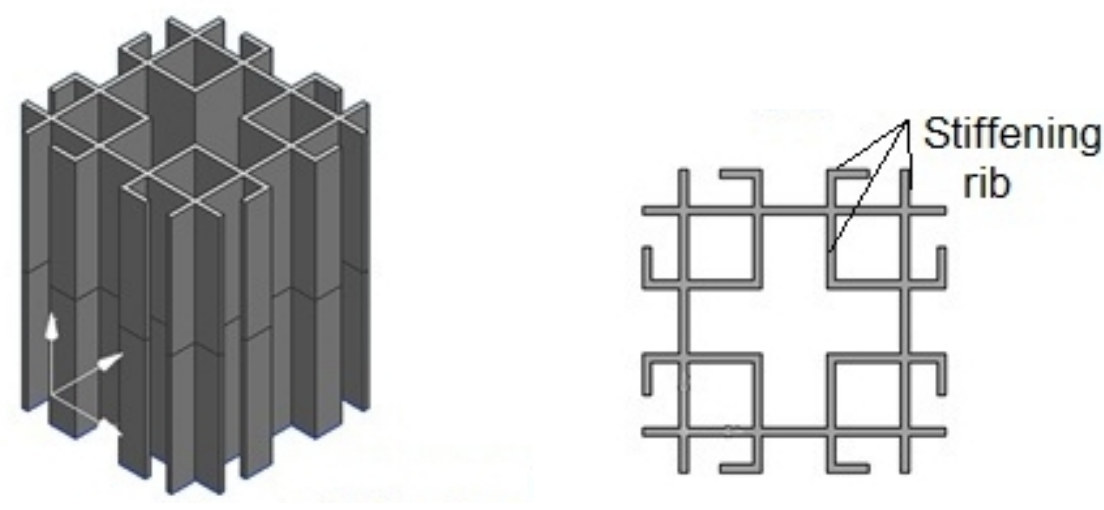

Fig.1 Sections

\section{Finite element model}

The stress-strain curve of aluminium alloy possesses nonlinearity and continuity, so their constitutive relationship cannot be simplified into the ideal elastic-plastic mode which is commonly used for steel. In order to make the analysis of aluminium alloy more accurate, the complicated model was used. The Ramberg-Osgood model, i.e. $\varepsilon=\sigma / E+0.002\left(\sigma / f_{0.2}\right)^{n}$, was the ideal model because it can well describe the constitutive relationship of aluminium alloys. The exponent $n$ can be calculated by Steinhardt proposal, i.e. $n=f_{0.2} / 10$ [3]. And the results display that mechanical properties of aluminium alloy components are closely associated with the value of $n$.

Additionally, a sort of simple and effective classification method which is suitable for the analysis of aluminium members was improved. The aluminium materials whose temper was T6 belong to weak hardening alloy, and the else belong to strong hardening alloy. Another classification method which is comparison of the size of the parameter $n$. when $n>20$, the aluminium alloy materials is weak hardening alloy, and the else is strong hardening alloy [4].

From the above analysis, 6061-T4 aluminium alloy and 6061-T6 aluminium alloy were selected. In addition, the Ramberg-Osgood model and the Steinhardt proposal were used in this article. Also the elastic modulus of aluminium alloy was set to $70000 \mathrm{MPa}$.

In this article, BEAM189 was used which is based on Timoshenko beam theory. In addition, the BEAM189 applied to nonlinear analysis of large deformation and large strain. Above all, when the BEAM189 was selected, the user could customize the section.

The load-deformation process of aluminium alloy components under axial compression were analyzed with ANSYS. Moreover, by adopting the analysis methods, the effect of the material non-linearity, geometric non-linearity and initial imperfection were considered. And the peak point of the load- deformation curve is the stability bearing capacity of the component. Note that the initial sinusoidal curvature was adopted as the geometrical imperfection, and the characteristic parameter of the displacement at mid-span was equal to $l / 1000$ ( $l$ being the length of component) [4]. The reason is that the influence of aluminium alloy extrusion profiles section size deviation is usually small. And when the initial bending of arch rise takes $l / 1000$, the extrusion residual stress value is very small. So the analysis can be neglected the residual stress and the non-uniform stress of mechanical properties effects on the bearing capacity.

\section{Finite element analysis}

Different section types possess different stability properties. The new section could only take place bending instability because of its warping constant and torsion constant are large. The typical failure modes of aluminium alloy members under axial compression were showed by Fig. 2 . 


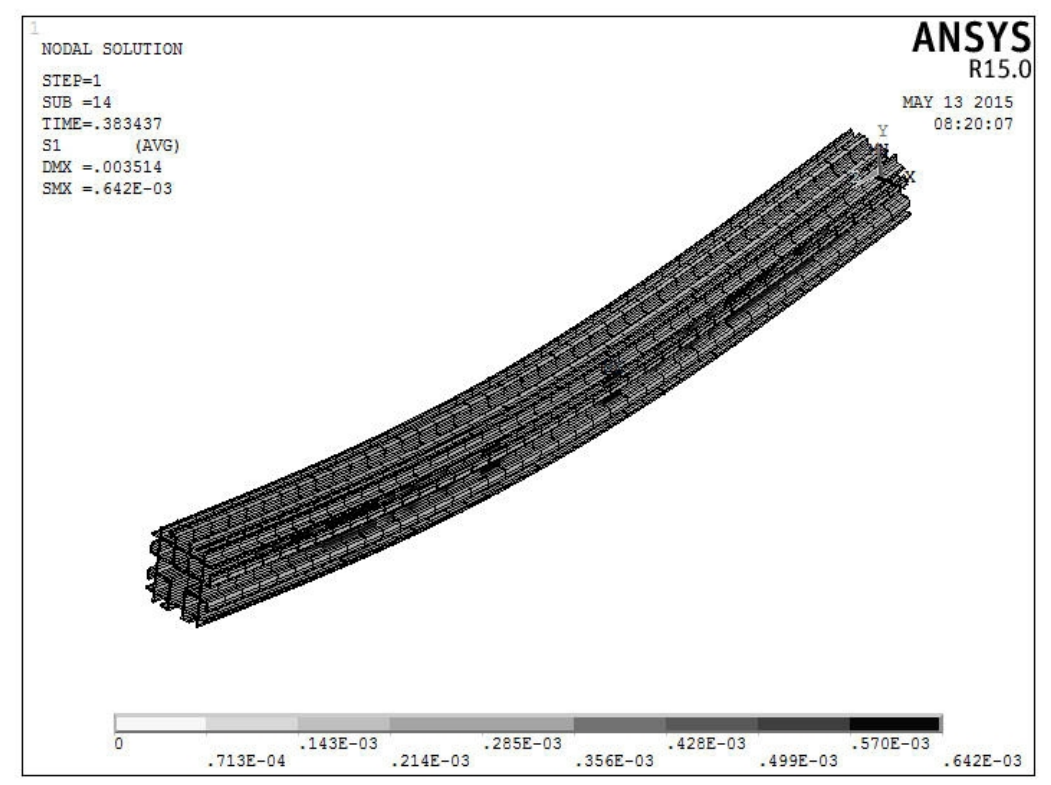

Fig.2 Typical failure modes

In order to study the different mechanical properties between new sections and $\mathrm{H}$-sections under the axial loading, load-deformation curves of new sections, H-section under major axis bending and $\mathrm{H}$-section under minor axis bending were calculated and compared. When the slenderness ratio was equal to 60, the load-deformation curves were showed by Fig. 3. It is important to note that the 6061-T4 aluminium was showed by (a) and the 6061-T6 aluminium was showed by (b). In addition, through the finite element analysis, the ultimate strengths also were got.

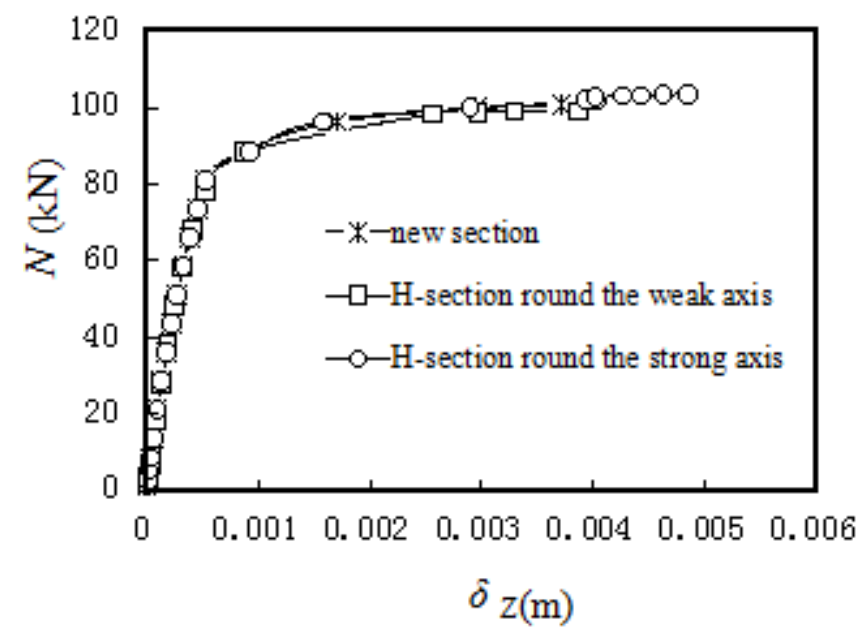

(a) 6061-T4 aluminium alloy

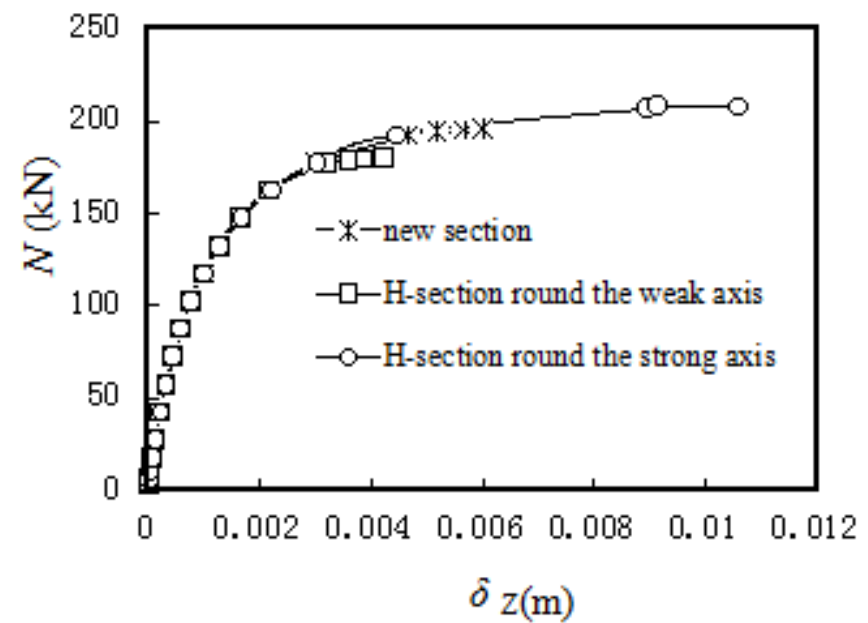

(b) 6061-T6 aluminium alloy

Fig. 3 Load-deformation curve

For comparison purposes, the areas of three kinds of specimens were set to exactly same, i.e., $0.001246 \mathrm{~m}^{2}$. The nominal yield strengths of 6061-T4 and 6061-T6 aluminium alloy were 110MPa and $240 \mathrm{MPa}$ [5], respectively. According to the calculation results, the slenderness ratio-stability factor curves were obtained, as shown by Fig.5. It is important to note that the 6061-T4 aluminium was showed by (a) and the 6061-T6 aluminium was showed by (b). 


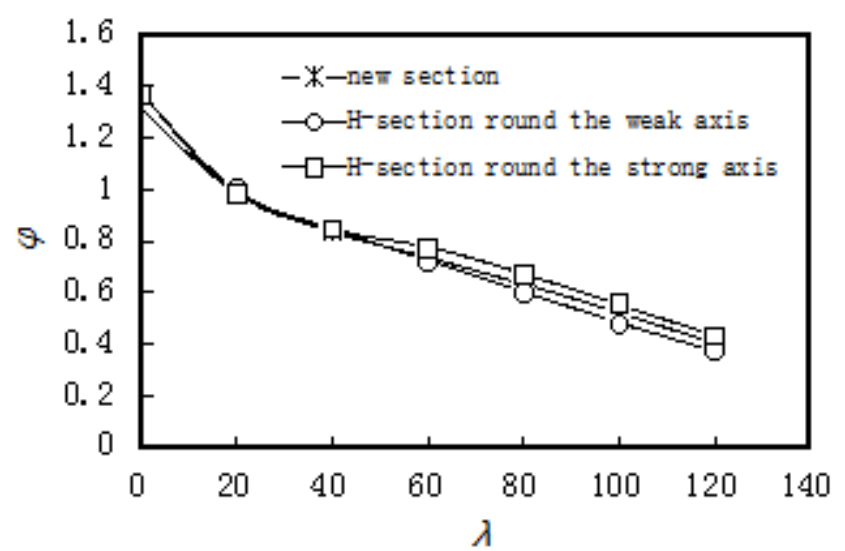

(a) 6061-T4 aluminium alloy

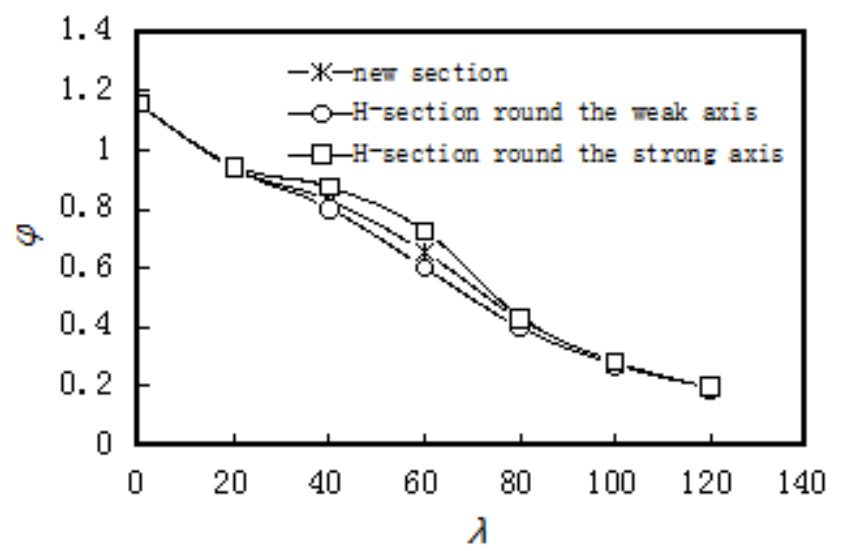

(b) 6061-T6 aluminium alloy

Fig. 4 The slenderness ratio-stability factor curves

From Fig. 4, it is found that the three kinds of specimens exhibit different characteristics when the slenderness ratio changes. The stability factor of the new section is between that of the strong axes of H-section and the weak axes of H-section. To the structural design, the stability factor of new section can be partially safe to select the weak axes of $\mathrm{H}$-section.

\section{Discussion}

The stable performance of new section of aluminium alloy members under axial compression had been analyzed in this paper. And the discussions are as follows:

(1) The constitutive parameters of aluminium alloy, such as nominal yield strength, elastic modulus and exponent $n$, have an important influence on their stable performance.

(2) For the same alloy types, with the increase of slenderness ratio, the variation trends of the load-deformation curves are similar. Results of new section agreed well with H-sections.

(3) The stability factors of the new section are between that of the strong axes of H-section and the weak axes of H-section. To the structural design, the stability factors of new section can be partially safe to use the factors of the weak axes of $\mathrm{H}$-section.

\section{Acknowledgements}

This work was financially supported by National Natural Science Foundation of China (51208109) and Program for Outstanding Young Scientific Talents in Fujian Province University (JA13209).

\section{References}

[1] X.N. Guo, Z.Y. Shen, Y.Q. Li, Z.G. Qiu, N.L. Yao: Journal of Building Structures, Vol. 28, No. 6 (2007). p. 118.

[2] J.H. Zhu, P. Wang, T.J. Liu: submitted to Journal of Building Structures (2014)

[3] M.M. Federico: Aluminium alloy structures (Chapman \& Hall, London 1995).

[4] Z. Zhang, J.L. Chen: Journal of Fujian University of Technology, Vol. 7, No.3 (2009). p. 217.

[5] National standards of P.R.C: Code for the design of aluminium structures GB 50429-2007. 